Luisa Agnello, Matteo Vidali, Bruna Lo Sasso, Rosaria Vincenza Giglio, Caterina Maria Gambino, Concetta Scazzone, Anna Maria Ciaccio, Giulia Bivona and Marcello Ciaccio*

\title{
Monocyte distribution width (MDW) as a screening tool for early detecting sepsis: a systematic review and meta-analysis
}

https://doi.org/10.1515/cclm-2021-1331

Received December 27, 2021; accepted February 8, 2022; published online February 15, 2022

\section{Abstract}

Objectives: Monocyte distribution has recently emerged as a promising biomarker of sepsis, especially in acute setting, such as Emergency Department and Intensive Care Unit. This study aimed to evaluate the accuracy of monocyte distribution width (MDW) for early detecting patients with sepsis by performing a systemic review and metaanalysis of published studies.

Methods: Relevant publications were identified by a systematic literature search on PubMed and Google Scholar from inception to September 07, 2021. Studies were divided into two groups based on the sepsis criteria applied, namely sepsis-2 or sepsis-3.

Results: Ten studies including 9,475 individuals, of whom 1,370 with sepsis (742 according Sepsis-2 and 628 according to Sepsis-3), met the inclusion criteria for our metaanalysis. The pooled sensitivity and specificity were

Luisa Agnello and Matteo Vidali contributed equally to this work.

*Corresponding author: Prof. Marcello Ciaccio, Institute of Clinical Biochemistry, Clinical Molecular Medicine and Clinical Laboratory Medicine, Department of Biomedicine, Neurosciences and Advanced Diagnostics, University of Palermo, Palermo, Italy; and Department of Laboratory Medicine, University Hospital

“P. Giaccone”, Palermo, Italy, E-mail: marcello.ciaccio@unipa.it Luisa Agnello, Concetta Scazzone and Giulia Bivona, Institute of Clinical Biochemistry, Clinical Molecular Medicine and Clinical Laboratory Medicine, Department of Biomedicine, Neurosciences and Advanced Diagnostics, University of Palermo, Palermo, Italy Matteo Vidali, Foundation IRCCS Ca' Granda Ospedale Maggiore Policlinico, Milan, Italy

Bruna Lo Sasso, Rosaria Vincenza Giglio and Caterina Maria Gambino, Institute of Clinical Biochemistry, Clinical Molecular Medicine and Clinical Laboratory Medicine, Department of Biomedicine, Neurosciences and Advanced Diagnostics, University of Palermo, Palermo, Italy; and Department of Laboratory Medicine, University Hospital “P. Giaccone”, Palermo, Italy

Anna Maria Ciaccio, University Hospital “P. Giaccone”, Palermo, Italy
0.789 and 0.777 for Sepsis-2 criteria, 0.838 and 0.704 for Sepsis-3 criteria.

Conclusions: MDW represents a reliable biomarker for sepsis screening.

Keywords: biomarker; monocyte distribution (MDW); monocytes; screening; sepsis.

\section{Introduction}

Sepsis represents an important health burden worldwide. Early recognition is fundamental for promptly starting the appropriate treatment in order to improve the patient's outcome and to reduce mortality. However, the diagnosis of sepsis, which relies on the integration of clinical and laboratory findings, is still challenging. Indeed, sepsis is characterised by no specific signs and symptoms. Additionally, blood culture, which represents the gold standard for sepsis diagnosis, has several drawbacks, including a long turnaround time and a high rate of false-negative findings (up to 70\%) [1]. Thus, many efforts are ongoing for identifying a reliable biomarker to early recognize patients at high risk of sepsis.

Recently, the monocyte distribution width (MDW) emerged as a valuable biomarker, allowing to identifying patients at risk of sepsis admitted to acute settings, such as the Emergency Department (ED) and Intensive Care Unit (ICU). MDW is a cell population data (CPD) parameter, which is rapidly and automatically calculated by lastgeneration DxH haematology analysers (Beckman Coulter, Inc.), along with routine complete blood cell (CBC) count. It reflects the heterogeneity in the size of circulating monocytes, which have a key role in the pathogenesis of sepsis since very early stages [2]. Indeed, monocytes represent the first-line defence against infection. After infectious stimuli, monocytes undergo activation leading to functional and morphological changes. Thus, during the early stages of infection and sepsis, the monocyte population is characterised by high heterogeneity, which can be detected by MDW [3]. Several Authors showed that an increased MDW 
is associated with an increased risk of sepsis. Additionally, the reference intervals and decisional values, two fundamental steps in the clinical validation process of a biomarker, have been established [2, 4]. Among all biomarkers of sepsis [5-7], MDW has a great potential to be introduced in clinical practice.

We performed a systematic review and comprehensive meta-analysis to evaluate the accuracy of MDW as a biomarker of sepsis.

\section{Materials and methods}

We followed the preferred reporting items for systematic reviews and meta-analysis (PRISMA) guidelines 2020 [8]. All studies investigating the diagnostic efficacy of MDW for sepsis were searched for inclusion.

\section{Literature search strategy}

Two reviewers systematically and independently (LA and MV) performed a comprehensive electronic search of PubMed and Google Scholar. The following Medical Subject Heading (MeSH) terms "MDW" and "sepsis" were used to search articles. No publication date restriction was applied, and the date of our search was until 07 September 2021.

\section{Study selection}

The inclusion criteria were: (i) retrospective and prospective study design; (ii) MDW measurement; (iii) evaluation of MDW for sepsis screening; (iv) the manuscript was written in the English language; (v) sufficient data were provided to calculate the outcome; (vi) inclusion of only adult patients (age $\geq 18$ years).

Exclusion criteria were: (i) evaluation of only the prognostic role of MDW; (ii) lack of evaluation of MDW accuracy; (iii) case reports, animal studies, reviews, and editorials (vi) other languages than English; (v) full-text not found.

\section{Data collection}

Two authors (LA and MV) independently collected data referring to study and patient characteristics. The extracted information from each study included first author name, year of publication, country, study design, clinical setting, sepsis criteria, study population (sample size and patients grouped according to sepsis- 2 or sepsis- 3 criteria), the tube used for blood collection, MDW cut-off value, outcome data [area under the roc curve (AUC), sensitivity, specificity, positive and negative predictive values].

\section{Statistical analysis}

Meta-analytical summaries of MDW performance were calculated following the bivariate binomial approach by fitting a generalized linear mixed model (GLMM) [9-11]. Summary pooled sensitivity, specificity, positive likelihood ratio, negative likelihood ratio and diagnostic odds ratio (DOR) were calculated by R Language v.4.0.3 (R Foundation for Statistical Computing, Vienna, Austria) and RStudio IDE v.1.3.1093 (RStudio, PBC, Boston, MA) with the lme4, mada and meta packages [12]. Heterogeneity across the studies was evaluated by plotting sensitivities and specificities, together with their 95\%CI, by Forest and Crosshair plots [13] and by inconsistency index (I2), calculated as $100 \%{ }^{\star}(Q-\mathrm{df}) / Q$, where $Q$ is Cochran's heterogeneity statistic and the degrees of freedom. Publication bias was evaluated by funnel plot and Deeks's test [14].

\section{Results}

\section{Study selection}

The process of study selection is schematically presented in the PRISMA flow diagram (Figure 1). A total of 570 articles (20 from PubMed and 550 from Google Scholar) were obtained. After the removal of 20 duplicates, 550 studies were retrieved. After screening the title and abstracts, 536 studies were excluded because they were literature review, case reports, abstracts, experimental studies on animals, website product information, they were performed only on paediatric population, they did not measure MDW, they did not evaluate the diagnostic accuracy of MDW for sepsis. The full text of 14 studies was further evaluated. Finally, a total of 10 studies, performed in different settings (Intensive Care Unit, Emergency Department or Infectious Diseases Unit) were included.

\section{Study characteristics and quality assessment}

Sepsis-2 or sepsis-3 criteria were applied, respectively, in 4 and in 6 studies. The ten studies correspond to nine independent published works, since in the work by Hausfater [15] the same 1,517 patients investigated were classified using sepsis-2 [16] or sepsis-3 criteria [17]. The main characteristics and diagnostic performances of the studies are reported in Table 1. The sample size of the studies included was between 82 and 2,215. For studies applying sepsis-2 criteria $(n=4)$, the cut-off ranged from 20.5 to 23.5 , with sensitivity and specificity ranging, respectively, from 0.645 to 0.920 and from 0.604 to 0.929 (Table 1). For studies applying sepsis-3 criteria $(\mathrm{n}=6)$, the cut-off ranged from 19.2 to 24.6 , with sensitivity and specificity ranging, respectively, from 0.669 to 0.957 and from 0.499 to 0.797 (Table 1). The forest plots and the crosshair plots for sensitivity and specificity across the studies, classified according to sepsis- 2 or sepsis-3 criteria, are reported in 


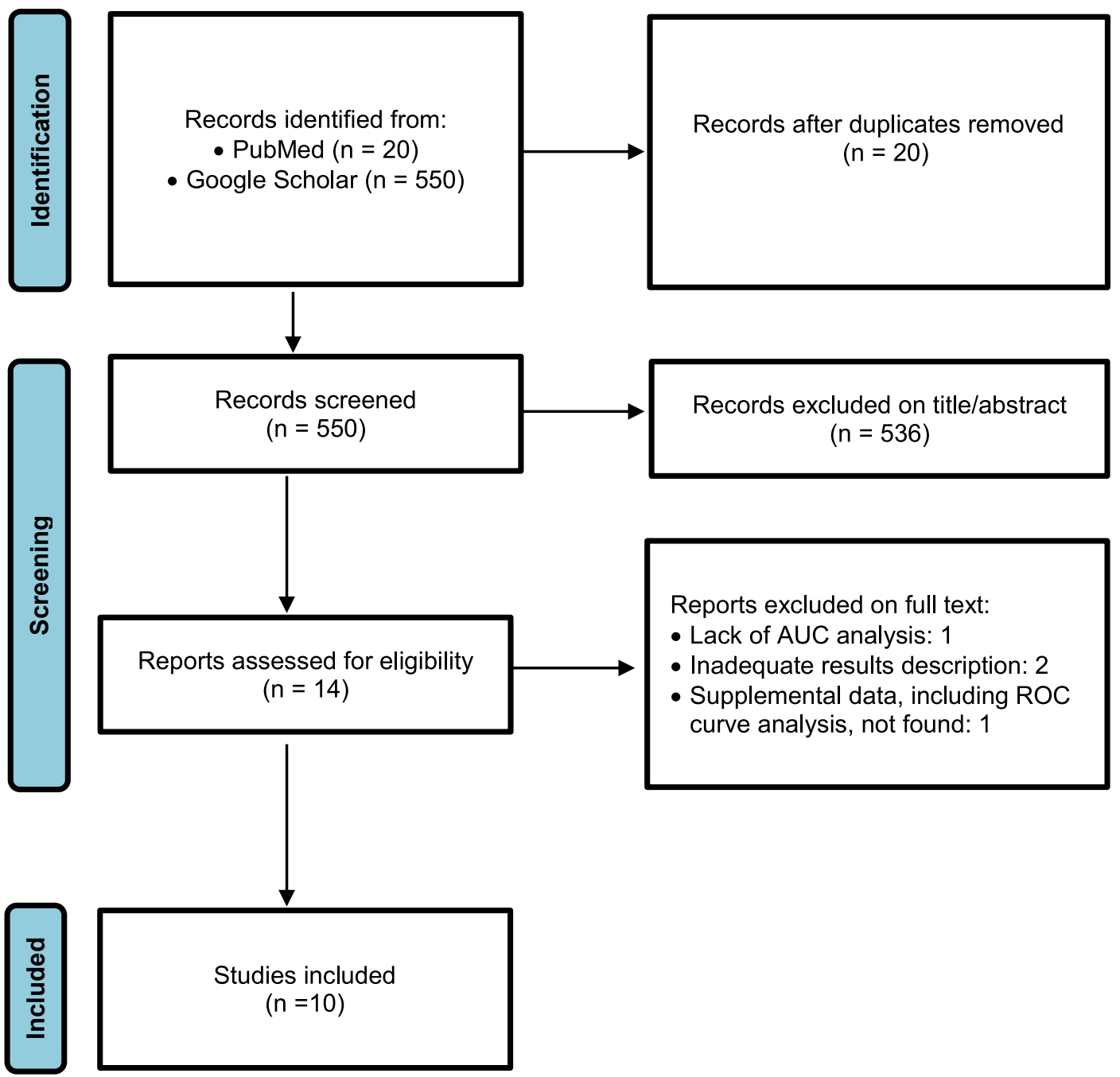

Figure 1: PRISMA 2020 study selection flow diagram.

Figures 2 and 3. The plots suggest high variability for both sensitivity and specificity. No publication bias was detected by inspection of funnel plot and formal Deeks's test.

\section{Diagnostic accuracy of MDW for sepsis}

Due to the significant heterogeneity observed in the sensitivity and specificity data (respectively, I2 87.9\% and 99.3\% for sepsis-2 studies, $81.7 \%$ and $93.6 \%$ for sepsis-3 studies), a random-effects model was applied. Meta-analytical summaries of MDW performances were obtained following a bivariate binomial method by fitting a generalized linear mixed model (GLMM) [11].

For sepsis-2 studies pooled results were as follows: sensitivity 0.789 (95\%CI $0.648-0.884)$, specificity 0.777 (95\%CI 0.613-0.884), positive likelihood ratio 3.533 (95\%
CI 1.666-7.493), negative likelihood ratio 0.271 (95\%CI 0.131-0.563) and DOR 13.032 (95\%CI 2.979-57.007).

For sepsis-3 studies pooled results were as follows: sensitivity 0.838 (95\%CI $0.740-0.904)$, specificity 0.704 (95\%CI 0.622-0.775), positive likelihood ratio 2.833 (95\% CI 2.166-3.705), negative likelihood ratio 0.230 (95\%CI 0.140-0.379) and DOR 12.312 (95\%CI 6.322-23.976).

\section{Discussion}

In this systematic review and meta-analysis, we evaluated the accuracy of MDW as a biomarker of sepsis by analysing results from ten studies, including a total of 9,475 individuals, of whom 1,370 had sepsis, 742 diagnosed according to Sepsis-2 criteria and 628 according to Sepsis-3 criteria. We included only studies performed on adult 


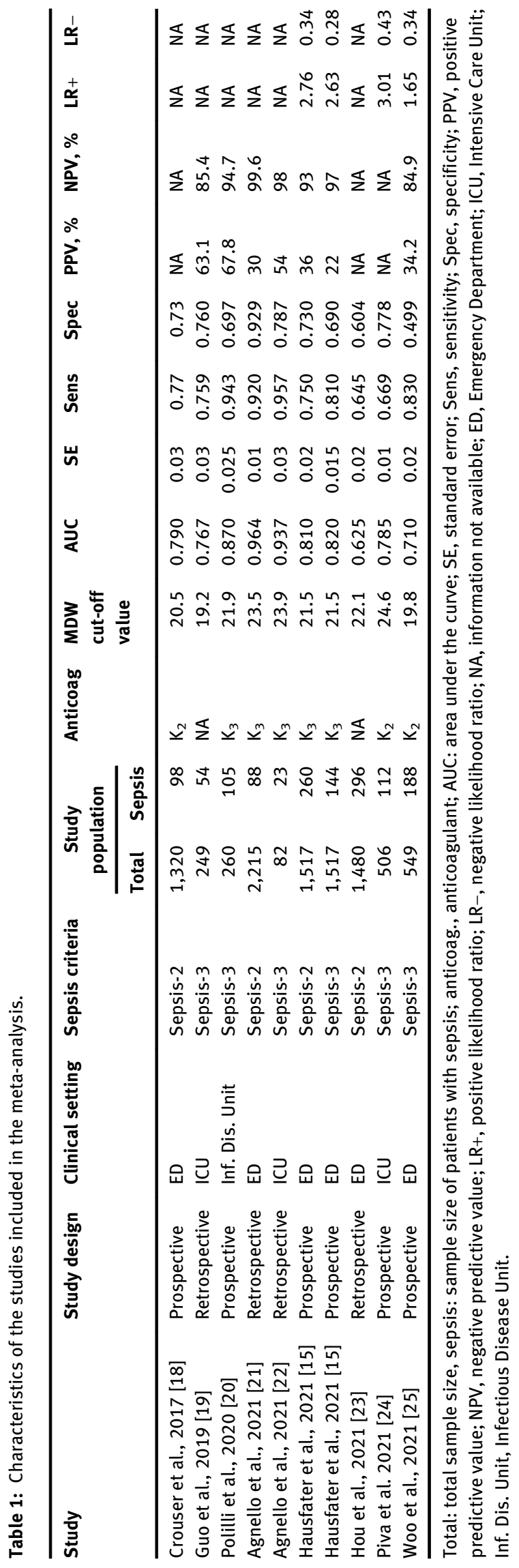

individuals, who were admitted to different clinical settings, including ED ( $\mathrm{n}=7)$, ICU ( $\mathrm{n}=2)$, and Unit of Infectious Diseases $(n=1)$. We excluded paediatric individuals and patients with COVID-19 in order to avoid bias. Indeed, although some Authors showed that COVID-19 patients have increased levels of MDW [26, 27], such disease is characterised by a high heterogeneity both a clinical and clinicalbiochemistry point of view. Additionally, COVID-19 patients show significant differences among the epidemiological waves. Thus, in order to avoid to further increasing the heterogeneity already found among studies, we considered it appropriate to exclude COVID-19 patients.

Finally, since sepsis-2 and sepsis-3 criteria identify patients with sepsis by using different parameters, we considered it appropriate to distinguish studies according to these criteria.

In all studies, MDW showed good diagnostic accuracy for early detecting patients with sepsis, with an AUC ranging from 0.625 to 0.964 . Due to different cut-offs reported in the published studies, we did not calculate weighted estimates of sensitivity and specificity for MDW at a common threshold, using sensitivity and specificity data at the different cut-offs. Instead, we estimated pooled summaries using a bivariate binomial approach. Specifically, we found pooled sensitivity and specificity of 0.789 and 0.777 , respectively, for sepsis- 2 criteria, and 0.838 and 0.704, respectively, for sepsis-3 criteria. DORs were, respectively, 13.032 and 12.312. Forest plot analysis showed significant heterogeneity among the included studies. However, we did not apply additional statistical methods to further investigate this heterogeneity due to the small number of studies. Thus, neither the meta-regression nor a subgroup analysis was performed. We can hypothesize that the main sources of heterogeneity could be the anticoagulant used for collecting blood samples ( $\mathrm{K}_{3}$-EDTA vs. $\mathrm{K}_{2}$-EDTA), the clinical wards where the studies were performed, the cut-off used and the characteristics of the study population. Specifically, the manufacturer has described the influence of the anticoagulant, with a 1.5 unit offset between $\mathrm{K}_{2^{-}}$and $\mathrm{K}_{3}$ - EDTA (https://www. beckmancoulter.com/download/file/wsr-262828/ C21894AC?type=pdf).

To the best of our knowledge, this is the first metaanalysis to assess the value of MDW as a biomarker of sepsis. Our findings show that MDW has high accuracy for detecting patients at risk of sepsis in different clinical wards. The introduction of MDW in clinical practice is very attractive because it has the great advantage of being a parameter belonging to the CBC. Thus, its evaluation is fast, easy, low-cost, and it does not require an additional blood sample, as for the measurement of other biomarkers 


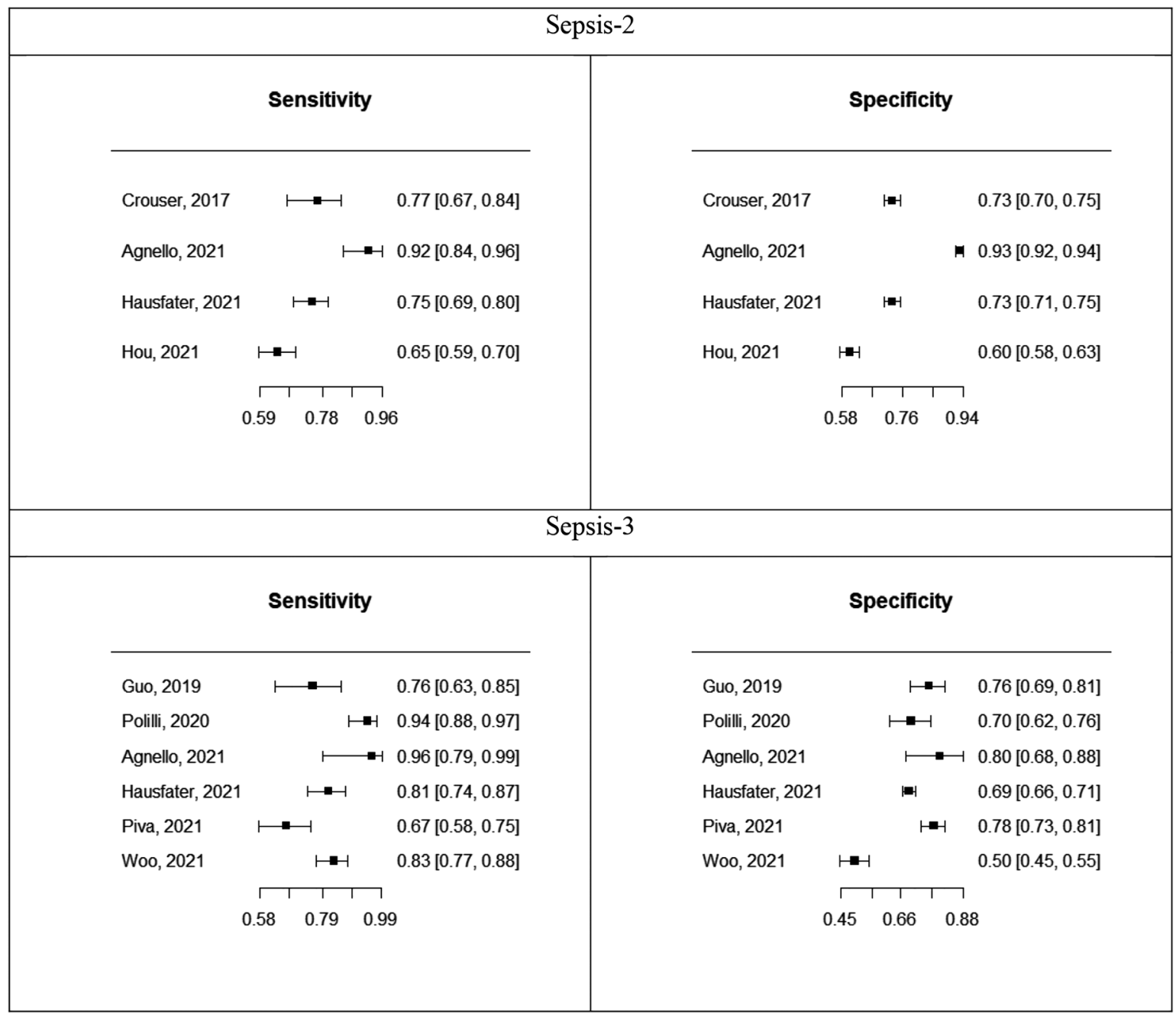

Figure 2: Forest plots of sensitivity (left column) and specificity (right column) of the studies investigated divided by the use of sepsis-2 (above) or sepsis-3 (below) criteria.

Studies were ordered following date of publication.

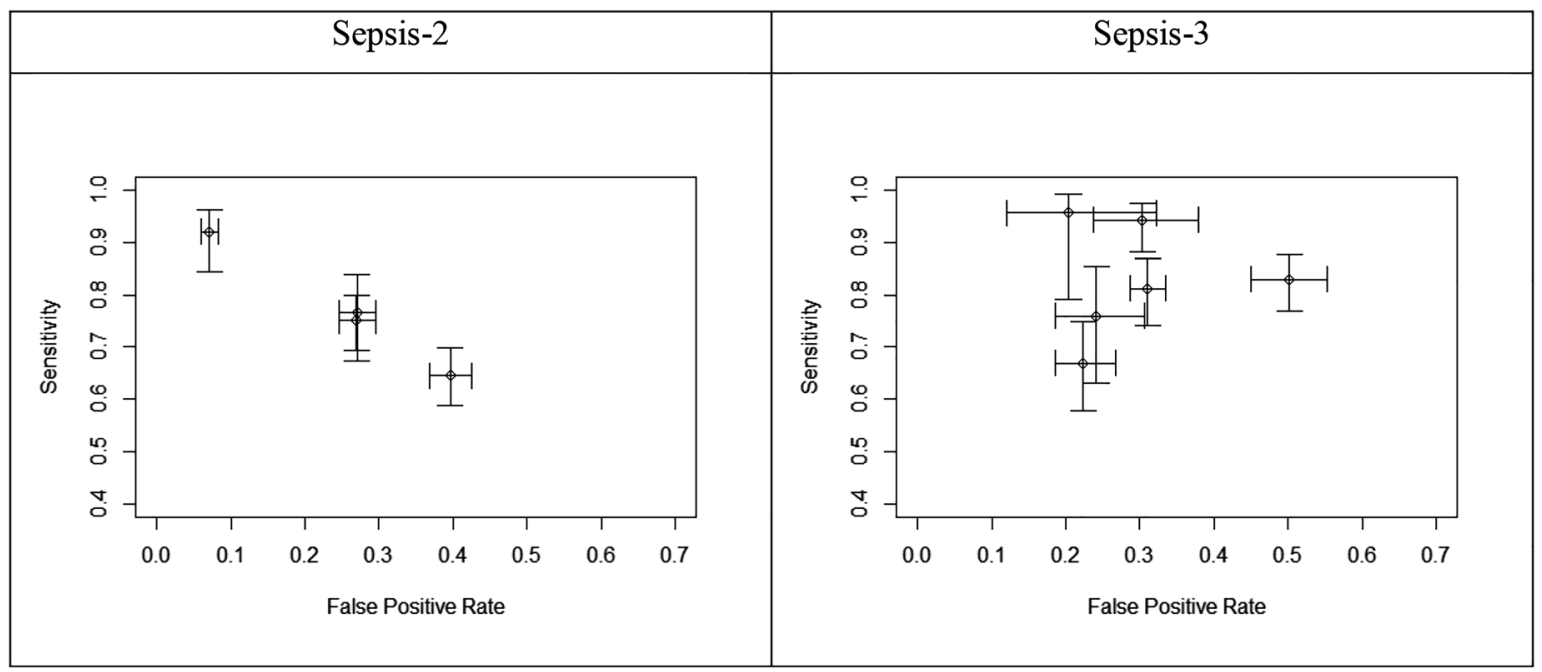

Figure 3: Crosshair plots of the sensitivity and specificity across the studies investigated divided by the use of sepsis-2 (left) or sepsis-3 (right) criteria.

Studies were ordered following date of publication. 
of sepsis. $\mathrm{CBC}$ is the most common laboratory test required in all patients admitted in any clinical ward, from ED to ICU [28]. Thus, it could be available to any clinicians in every moment of the patient's care path, also when there is no suspect of sepsis. On the contrary, the most commonly used biomarkers of sepsis, such as C-reactive protein and procalcitonin, are ordered by clinicians in an advanced stage, when there is the clinical suspicion of sepsis.

An altered MDW value should be interpreted as a "red flag" for sepsis and the clinicians should monitor the patient and further investigate to confirm the suspect of sepsis. Noteworthy, several Authors reported that MDW has a high negative predictive value [15, 20-22]. Thus, a value of MDW below the cut-off should exclude with high reliability sepsis.

\section{Conclusions}

This meta-analysis showed that MDW has high accuracy in early detecting patients with sepsis. Thus, it could represent a reliable tool for guiding clinicians in the appropriate management of patients, also when sepsis is not suspected.

Research funding: None declared.

Author contribution: All authors have accepted responsibility for the entire content of this manuscript and approved its submission.

Competing interests: Authors state no conflict of interest. Informed consent: Not applicable.

Ethical approval: Not applicable.

\section{References}

1. Tan M, Lu Y, Jiang H, Zhang L. The diagnostic accuracy of procalcitonin and $\mathrm{C}$-reactive protein for sepsis: a systematic review and meta-analysis. J Cell Biochem 2019;120:5852-9.

2. Agnello L, Lo Sasso B, Vidali M, Scazzone C, Gambino CM, Giglio RV, et al. Validation of monocyte distribution width decisional cutoff for sepsis detection in the acute setting. Int J Lab Hematol 2021;43: 0183-5.

3. Radzyukevich YV, Kosyakova NI, Prokhorenko IR. Participation of monocyte subpopulations in progression of experimental endotoxemia (EE) and systemic inflammation. J Immunol Res 2021;2021:1762584.

4. Agnello L, Lo Sasso B, Bivona G, Gambino CM, Giglio RV, lacolino G, et al. Reference interval of monocyte distribution width (MDW) in healthy blood donors. Clin Chim Acta 2020;510:272-7.

5. Kataria Y, Remick D. Sepsis biomarkers. Methods Mol Biol 2021; 2321:177-89.
6. Bellia C, Agnello L, Lo Sasso B, Bivona G, Raineri MS, Giarratano A, et al. Mid-regional pro-adrenomedullin predicts poor outcome in non-selected patients admitted to an intensive care unit. Clin Chem Lab Med 2019;57:549-55.

7. Agnello L, Bivona G, Parisi E, Lucido GD, lacona A, Ciaccio AM, et al. Presepsin and midregional proadrenomedullin in pediatric oncologic patients with febrile neutropenia. Lab Med 2020;51: 585-91.

8. Page MJ, McKenzie JE, Bossuyt PM, Boutron I, Hoffmann TC, Mulrow CD, et al. The PRISMA 2020 statement: an updated guideline for reporting systematic reviews. PLoS Med 2021;18: e1003583.

9. Hamza TH, van Houwelingen HC, Stijnen T. The binomial distribution of meta-analysis was preferred to model withinstudy variability. J Clin Epidemiol 2008;61:41-51.

10. Jackson D, Law M, Stijnen T, Viechtbauer W, White IR. A comparison of seven random-effects models for meta-analyses that estimate the summary odds ratio. Stat Med 2018;37:1059-85.

11. Chu H, Cole SR. Bivariate meta-analysis of sensitivity and specificity with sparse data: a generalized linear mixed model approach. J Clin Epidemiol 2006;59:1331-2.

12. Software for meta-analysis of DTA studies. Available from: https://methods.cochrane.org/sdt/software-meta-analysis-dtastudies [Accessed 5 Feb 2022].

13. Phillips B, Stewart LA, Sutton AJ. 'Cross hairs' plots for diagnostic meta-analysis. Res Synth Methods 2010;1:308-15.

14. van Enst WA, Ochodo E, Scholten RJ, Hooft L, Leeflang MM. Investigation of publication bias in meta-analyses of diagnostic test accuracy: a meta-epidemiological study. BMC Med Res Methodol 2014;14:70.

15. Hausfater P, Robert Boter N, Morales Indiano C, Cancella de Abreu M, Marin AM, Pernet J, et al. Monocyte distribution width (MDW) performance as an early sepsis indicator in the emergency department: comparison with CRP and procalcitonin in a multicenter international European prospective study. Crit Care 2021;25:227.

16. Levy MM, Fink MP, Marshall JC, Abraham E, Angus D, Cook D, et al. 2001 SCCM/ESICM/ACCP/ATS/SIS international sepsis definitions conference. Intensive Care Med 2003;29:530-8.

17. Singer M, Deutschman CS, Seymour CW, Shankar-Hari M, Annane $D$, Bauer $M$, et al. The third international consensus definitions for sepsis and septic shock (Sepsis-3). JAMA 2016; 315:801-10.

18. Crouser ED, Parrillo JE, Seymour C, Angus DC, Bicking K, Tejidor L, et al. Improved early detection of sepsis in the ED with a novel monocyte distribution width biomarker. Chest 2017;152:518-26.

19. Guo F, Feng YC, Zhao G, Wu HL, Xu L, Zhao J, et al. The leukocyte VCS parameters compared with procalcitonin, interleukin- 6 , and soluble hemoglobin scavenger receptor SCD163 for prediction of sepsis in patients with cirrhosis. Dis Markers 2019;2019: 1369798.

20. Polilli E, Sozio F, Frattari A, Persichitti L, Sensi M, Posata R, et al. Comparison of monocyte distribution width (MDW) and procalcitonin for early recognition of sepsis. PLoS One 2020;15: e0227300.

21. Agnello L, Bivona G, Vidali M, Scazzone C, Giglio RV, Iacolino G, et al. Monocyte distribution width (MDW) as a screening tool for 
sepsis in the emergency department. Clin Chem Lab Med 2020; 58:1951-7.

22. Agnello L, Sasso BL, Giglio RV, Bivona G, Gambino CM, Cortegiani A, et al. Monocyte distribution width as a biomarker of sepsis in the intensive care unit: a pilot study. Ann Clin Biochem 2021;58:70-3.

23. Hou SK, Lin HA, Chen SC, Lin CF, Lin SF. Monocyte distribution width, neutrophil-to-lymphocyte ratio, and platelet-tolymphocyte ratio improves early prediction for sepsis at the emergency. J Personalized Med 2021;11:732.

24. Piva E, Zuin J, Pelloso M, Tosato F, Fogar P, Plebani M. Monocyte distribution width (MDW) parameter as a sepsis indicator in intensive care units. Clin Chem Lab Med 2021;59:1307-14.
25. Woo A, Oh DK, Park CJ, Hong SB. Monocyte distribution width compared with C-reactive protein and procalcitonin for early sepsis detection in the emergency department. PLoS One 2021; 16:e0250101.

26. Riva G, Nasillo V, Luppi M, Tagliafico E, Trenti T. Linking COVID-19, monocyte activation and sepsis: MDW, a novel biomarker from cytometry. EBioMedicine 2021;75:103754.

27. Lippi G, Sanchis-Gomar F, Henry BM. Pooled analysis of monocyte distribution width in subjects with SARS-CoV-2 infection. Int J Lab Hematol 2021;43:0161-0163.

28. Agnello L, Giglio RV, Bivona G, Scazzone C, Gambino CM, lacona A, et al. The value of a complete blood count (CBC) for sepsis diagnosis and prognosis. Diagnostics 2021;11:1881. 